\title{
Project RISARD
}

\section{Marcin P. Gawroński*, Krzysztof Goździewski, Krzysztof Katarzyński}

Centre for Astronomy, Faculty of Physics, Astronomy and Informatics, Nicolaus Copernicus

University, Grudziadzka 5, 87-100 Toruń, Poland

E-mail: motylekeastro.umk.pl*

Red Dwarfs (RDs) are the most common, low-mass stars $\left(\lesssim 0.5 \mathrm{M}_{\odot}\right)$ in the Solar neighbourhood, and probably in the Universe as well. Most (likely all) young RDs are magnetically active, and therefore it is impossible to measure their radial velocities (RVs) with the accuracy required by contemporary planetary searches $(5-50 \mathrm{~m} / \mathrm{s})$. Some of RDs are known as a source of variable radio emission at centimetre wavelengths. This radiation is relatively weak ( $\sim 0.2$ to $1 \mathrm{mJy})$. However, recent development of the EVN and e-VLBI systems in terms of sensitivity gives a new possibility to investigate such stars. Here, we present first results from our ongoing RISARD project (Radio Interferometric Survey of Active Red Dwarfs). The main goal of this project is to detect an exoplanet by direct, precise measurements of a RD position and possible changes to this position caused by the planet.

11th European VLBI Network Symposium \& Users Meeting,

October 9-12, 2012

Bordeaux, France

${ }^{*}$ Speaker. 


\section{Introduction}

It is believed that Red Dwarfs (RDs) are the most numerous stars in the Universe ( $>70 \%)$. These low-mass $\left(\lesssim 0.5 \mathrm{M}_{\odot}\right)$ objects constitute also more than $40 \%$ of total stellar mas in our Galaxy [7]. However, it is still unclear what is the formation rate of planets (or low-mass companions) around these very common object. Is this rate similar, less or higher to the rate that we observe for solar-type FGK stars? RDs with their low-masses are very attractive targets for planetary search because in principle it should be possible to detect their companions down to the super-Earth masses.

The recent and ongoing RV surveys are focused on RDs samples that are necessarily biased towards chromospherically quiet and old objects. For instance, the RV survey of M-dwarfs with the VLT+UVES already includes a brown dwarf desert object and several low-mass companion candidates around early-type M-dwarfs [9]. A remarkable multi-planet resonant system was discovered around the M-dwarf Gliese 876, hosting two Jupiters [10]. The M-dwarfs are also intensively monitored by the Geneva Planet Search Team (e.g. [11]) that reported a multiple system of six planets around Gliese 581.

The currently known mass function of planetary candidates around RDs suggests that their jovian companions are less frequent than in the neighbourhood of the FGK-type stars. Especially the lack of so-called hot Jupiters is very striking. However, planets in the Neptune-mass range may be much more common (see Fig. 11). This is indicated by the recent discoveries from the RV surveys (e.g. [11]) of many multiple super-Earth systems around quiet M-dwarfs. Also, regarding solarlike dwarfs, the KEPLER transit detections indicate an exponential mass function of short-period planets in that mass range. This statistic is biased towards an old stars and it has been suggested [8] that this could be affected by the expected correlation between the occurrence rate of planetary systems and the metallicity of host stars [13]. It has been also pointed out that M-dwarfs hosting planets appear systematically metal-rich [8].

Magnetically active young M-dwarfs are producing a weak, variable radio emission at radio wavelengths [5]. It is believed that the electron cyclotron maser instability [6] and/or gyromagnetic emission (e.g. [4]) are responsible for this radiation. The VLBI technique has been already successfully used for the observations of low-mass active M-dwarfs [12] or even brown dwarfs [3]. The recent development of the EVN and e-VLBI systems improved significantly the sensitivity and opened a new observational window for stellar astrophysics. Current capabilities of European VLBI Network allow for observations of very weak $(\sim 100 \mu \mathrm{Jy})$, compact radio sources with the brightness temperature in the range of $10^{6}-10^{7} \mathrm{~K}$ like active RDs. This gives a new opportunity for planetary search in terms of an independent and very precise astrometric surveys.

\section{Project RISARD}

RISARD is a new astrometric project with EVN started in 2009. This project is dedicated for observations of very young $(\lesssim 1 \mathrm{Gyr})$ low-mass stars. The selection of young targets increases the probability of planetary system occurrence because of increased metallicity [13]. We attempt to reach a region in the physical and parameter space that cannot be explored by the RVs or transit photometry (due to orbital orientation bias and/or chromospheric activity). Moreover, the astrometric method may detect the stellar wobble around the barycentre independently of the orbital 


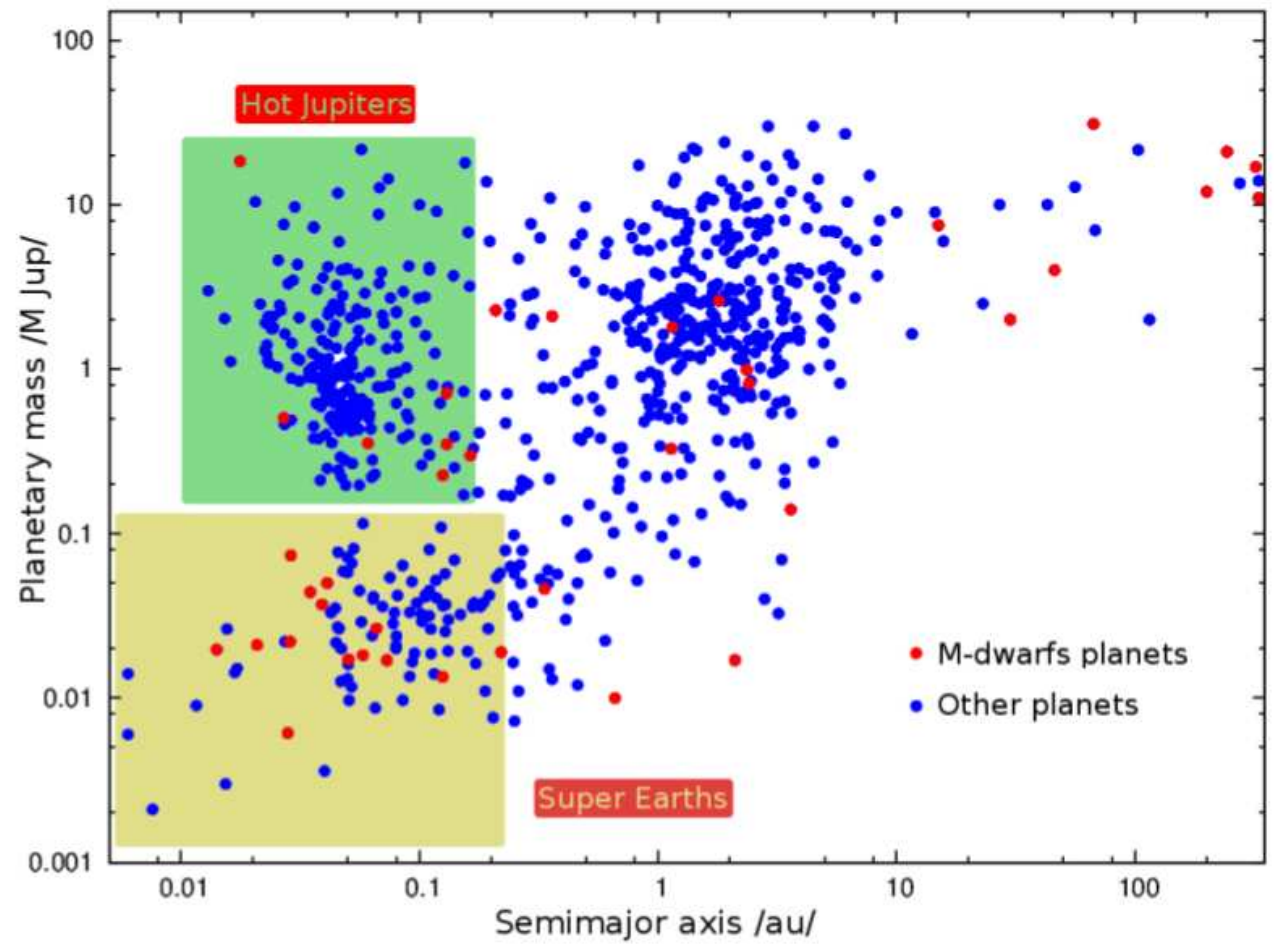

Figure 1: Planetary systems statistics based on The Extrasolar Planets Encyclopaedia (http://exoplanet.eu/). The M-dwarf planets are marked with red dots, the other planets with blue dots. An excess of Super-Earths in the case of RDs in comparison to the other stars is clearly visible.

inclination. As the final outcome, we expect to obtain non-biased statistics of low-mass companions around young M-dwarfs, and to estimate their occurrence rate. The mass function in the Solar neighbourhood exhibits a curious brown dwarf (BD) desert. Recent discoveries suggest that BDs may tend to form close to the low-mass stars. The astrometric signal of BDs should be easily detectable. Hence, even a lack of detections may contribute to the statistics of the sample of active, young RDs by setting upper limits to the masses of their putative companions. We stress that our survey complements ongoing RIPL survey with the help of the VLBA [2]. RIPL is a program conducted at $4 \mathrm{~cm}$ wavelength, initiated in 2007 that is focused on 29 active RDs within distance $\sim 10 \mathrm{pc}$ from Sun.

\section{Observations}

In the past three years, we have carefully prepared two test experiments to check the astrometric capabilities of the western EVN. First, we tested the sensitivity level and the astrometric accuracy (EG046, $7 \mathrm{hrs)}$ ) and after that we conducted $48 \mathrm{hrs}$ of e-VLBI observations (EG053) to select a new sample of radio active RDs within the distance $10 \lesssim d \lesssim 15$ pc from $\operatorname{Sun}^{1}$. The test observations EG046 has fully confirmed our expectation of the relative astrometric accuracy $\sim 0.1-0.3$ mas. In the experiment EG053 we observed 17 single, wide binary and multiple active

\footnotetext{
${ }^{1}$ The distance range has been chosen in order to not overlap with the RIPL sample.
} 

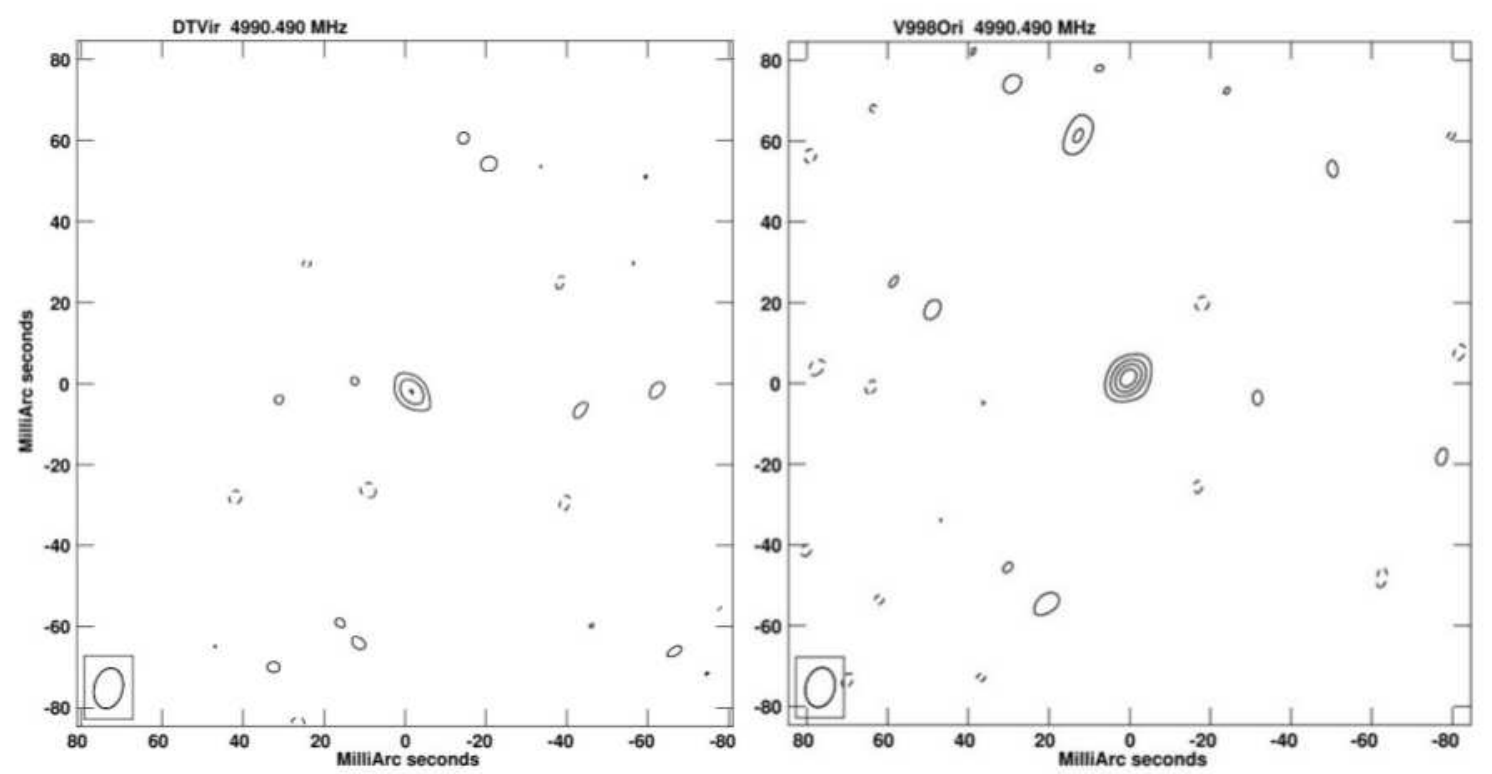

Figure 2: Radio maps of two selected active RDs. Left: DT Vir, right: V998 Ori.

red and brown dwarfs. The expected level of the radio emission of these stars was $\gtrsim 50 \mu \mathrm{Jy}$. We estimated this level from the X-ray-radio luminosity correlation (e.g. [1]). In addition we focused on the objects with declination $\gtrsim 0$. Preliminary results show the we have detected 12 RDs from our initial EG053 sample. In the Fig. 目 we show radio maps of two detected RDs.

Due to a proximity of selected stars to Sun, their high proper motions $(0.1-0.4 \mathrm{mas} / \mathrm{hr})$ and physical sizes $(\lesssim 0.5 \mathrm{mas})$ constitute a challenge to accurate astrometry. The observations of a given target should not last longer than $1.5 \mathrm{hrs}$. Our experience gained thanks to the initial EG046 and EG053 phase shows that $\sim 2$ hrs observation with phase-referencing ( $1.5 \mathrm{hrs}$ on the target + secondary calibrator) per target and 1 Gbps recording is sufficient to achieve an RMS at the level of $\simeq 25 \mathrm{mJy} /$ beam and hence avoid any problems caused by proper motions of stars. During 2012 we have started regular astrometric observations of $12 \mathrm{RDs}$ left in our sample. Second phase of RISARD consisted of 3-4 epochs of observations for each target. Observations are finished and data are currently reduced. After that phase we will re-evaluate again our sample and for the final 3-year part of RISARD only RDs with detection rate $\gtrsim 75 \%$ will remain in the sample.

Based on the initial results we have simulated astrometric observations for the purpose of RISARD project. Our calculations show that planets within the Jupiter-Saturn mass range will be detectable, and a favourable sampling of the relative orbit would make it possible to determine the Keplerian elements already from 10 observational epochs (note that in this example, we assume the astrometric parameters pre-determined). An example simulation for one of stars from RISARD sample is illustrated in Fig. 3. We present there also detection limits for putative low-mass components for another our target. We plan to extend RISARD survey in the future and estimate the dynamical masses of active RDs in binary systems placed within $20 \mathrm{pc}$ from Sun. 

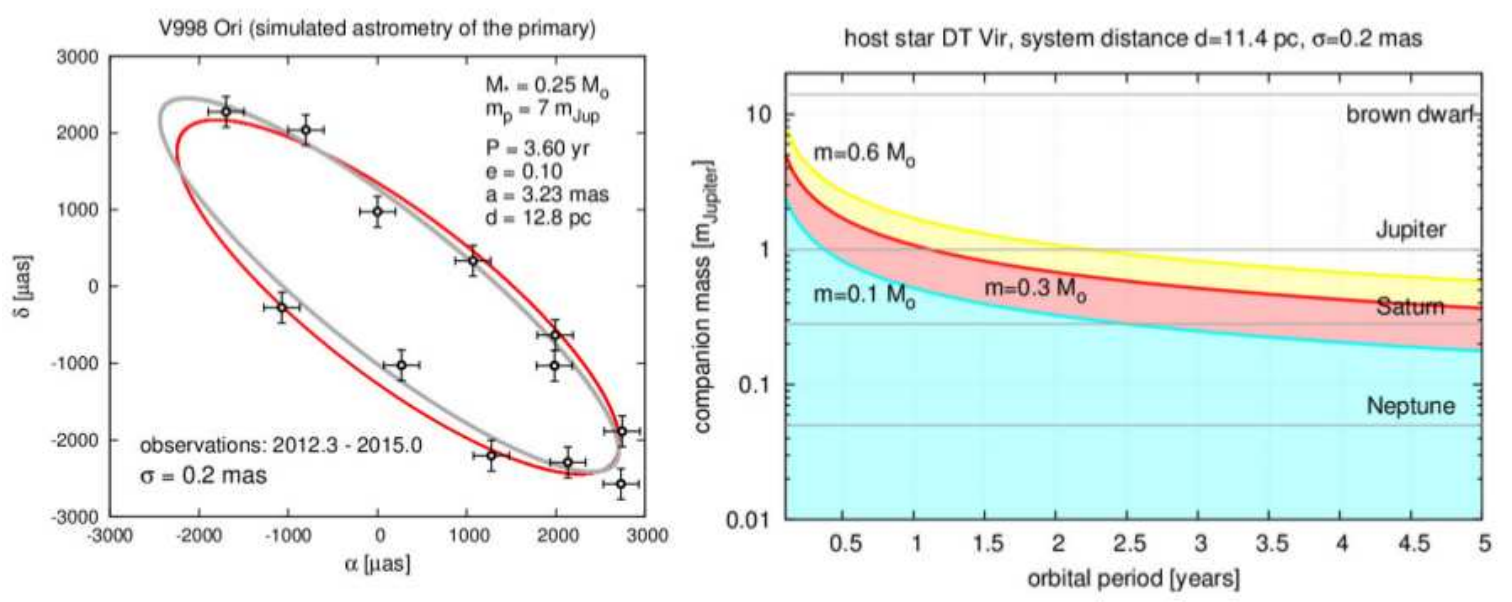

Figure 3: Simulations of observations and the detection limits based on results from experiments EG046 and EG053. Left: A simulation of an astrometric signal from $7 \mathrm{M}_{J u p}$ planet with the $3.6 \mathrm{yr}$ orbital period around V998 Ori. The mass of star has been assumed $0.25 \mathrm{M}_{\odot}$, the distance $12.8 \mathrm{pc}$ and the eccentricity of orbit $e$ $=0.01$. Red curve represents real orbit, grey - orbit estimated with the use of the simulated observations. Right: The estimated detection limits for DT Vir. Calculations have been done for three different dynamical masses of DT Vir. For the mass of host star in the range $0.1-0.3 \mathrm{M}_{\odot}$ RISARD will be able to detect planets in the Saturn-mass range on the $>4$ yrs orbit. In both simulation the error of relative astrometry has been assumed $\sigma=0.2$ mas.

\section{Acknowledgement}

We are grateful to Polish National Science Centre for their support of project RISARD (grant no. 2011/01/D/ST9/00735). The EVN is a joint facility of European, Chinese, South African, and other radio astronomy institutes funded by their national research councils.

\section{References}

[1] E. Berger, G. Basri, T.A. Fleming, et al., Simultaneous Multi-Wavelength Observations of Magnetic Activity in Ultracool Dwarfs. III. X-ray, Radio, and Ho Activity Trends in M and L dwarfs, ApJ 709 (2010) 332

[2] G.C. Bower, A. Bolatto, E.B. Ford, P. Kalas, Radio Interferometric Planet Search. I. First Constraints On Planetary Companions For Nearby, Low-Mass Stars From Radio Astrometry, ApJ 701 (2009), 1922

[3] J. Forbrich \& E. Berger, The First VLBI Detection of an Ultracool Dwarf: Implications for the Detectability of Sub-Stellar Companions, ApJ 706 (2009) 205

[4] M. Güdel, Stellar Radio Astronomy: Probing Stellar Atmospheres from Protostars to Giants, ARA\&A 40 (2002) 217

[5] M. Güdel, J.H.M. Schmitt, J.A. Bookbinder, T.A. Fleming, A tight correlation between radio and $X$-ray luminosities of M dwarfs, ApJ 415 (1993) 236

[6] G. Hallinan, A. Antonova, J.G. Doyle, et al., Confirmation of the Electron Cyclotron Maser Instability as the Dominant Source of Radio Emission from Very Low Mass Stars and Brown Dwarfs, ApJ 684 (2008) 644 
[7] T.J. Henry, Suspicious Characters Lurking in the Solar Neighborhood, in proceedings of Brown dwarfs and extrasolar planets, eds.: R. Rebolo, E.L. Martin, M.R. Zapatero Osorio, ASP Conference Series 13 (1998) 28

[8] J.A. Johnson \& K. Apps, On the Metal Richness of M Dwarfs with Planets, ApJ 699 (2009) 933

[9] M. Kürster, M. Zechmeister, M. Endl, The M dwarf planet search program with the VLT+UVES, in proceedings of Cool stars, stellar systems and the Sun, AIP Conference Proceedings 1094 (2009) 457

[10] G.W. Marcy, R.P. Butler, S.S. Vogt, D. Fisher, J.J. Lissauer, A Planetary Companion to a Nearby M4 Dwarf, Gliese 876, ApJ 505 (1998) 147

[11] M. Mayor, X. Bonfils, T. Foreveille, et al., The HARPS search for southern extra-solar planets. XVIII. An Earth-mass planet in the GJ 581 planetary system, A\&A 509 (2009) 487

[12] M.R. Pestalozzi, A.O. Benz, J.E. Conway, M. Güdel, VLBI observations of two single dMe stars: spatial resolution and astrometry, A\&A 353 (2000) 569

[13] N.C. Santos, G. Isreaelian, M. Mayor, Spectroscopic [Fe/H] for 98 extra-solar planet-host stars. Exploring the probability of planet formation, A\&A 415 (2004) 1153 